\title{
SOME RESULTS CONCERNING EXPONENTIAL DIVISORS
}

\author{
PETER HAGIS, JR.
}

Mathematics Department

Temple University

Philadelphia, PA 19122

(Received November 18, 1986 and in revised form February 9, 1987)

ABSTRACT. If the natural number $n$ has the canonical form $p_{1}{ }_{1}{ }_{p_{2}}{ }_{2} \ldots p_{r}{ }^{a}$ then $d=p_{1}{ }_{1}{ }_{1}{ }^{b}{ }_{2} \cdots p_{r}{ }_{r}$ is said to be an exponential divisor of $n$ if $b_{i} \mid a_{i}$ for $i=1,2, \ldots, r$. The sum of the exponential divisors of $n$ is denoted by $\sigma^{(e)}(n)$. $n$ is said to be an e-perfect number if $\sigma^{(e)}(n)=2 n ;(m ; n)$ is said to be an e-amicable pair if $\sigma^{(e)}(m)=m+n=\sigma^{(e)}(n) ; n_{0}, n_{1}, n_{2}, \ldots$ is said to be an e-aliquot sequence if $n_{i+1}=\sigma^{(e)}\left(n_{i}\right)-n_{i}$. Among the results established in this paper are: the density of the e-perfect numbers is .0087 ; each of the first 10,000,000 e-aliquot sequences is bounded.

KEYS WORDS AND PHRASES. Exponential divisors, e-perfect numbers, e-amicable numbers, e-aliquot sequences.

1980 AMS SUBJECT CLASSIFICATION CODE. 10A20.

1. INTRODUCTION.

If $\mathrm{n}$ is a positive integer greater than one whose prime-power decomposition is given by

$$
\mathrm{n}=\mathrm{p}_{1}{ }_{1} \mathrm{p}_{2}^{\mathrm{a}}{ }_{2} \ldots \mathrm{p}_{\mathrm{r}}^{\mathrm{a}} \mathrm{r}
$$

then $d$ is said to be an "exponential divisor" of $n$ if $d=p_{1}{ }_{1} p_{2}{ }^{b}{ }_{2} \ldots p_{r}{ }_{r}$ where $b_{i} \mid a_{i}$ for $i=1,2, \ldots, r$. The sum of all of the exponential divisors of $n$ is denoted by $\sigma^{(e)}(n)$. This function was first studied by Subbarao [1] who also initiated the study of exponentially perfect (or e-perfect) numbers.

The positive integer $n$ is said to be an e-perfect number if $\sigma^{(e)}(n)=2 n$. If $\sigma^{(e)}(n)=k n$, where $k$ is an integer which exceeds $2, n$ is said to be an e-multiperfect number. The properties of e-perfect and e-multiperfect numbers have been investigated by Straus and Subbarao [2] and Fabrykowski and Subbarao [3]. It has been proved, for example, that all e-perfect and e-multiperfect numbers are even. Also, if $\mathrm{n}$ is an e-perfect number and $3 / \mathrm{n}$ then $2^{110} / \mathrm{n}$ and $\mathrm{n}>10^{618}$.

While it is easy to show that there are an infinite number of e-perfect numbers, whether or not any e-multiperfect numbers exist is still an open question. Subbarao, Hardy and Aiello [4] have conjectured that there are no e-multiperfect numbers. They have proved that any which exist are very large. 
In Section 2 of the present paper the density of the set of e-perfect numbers is investigated. Section 3 is devoted to a study of e-amicable pairs, integers $m$ and $n$ such that $\sigma^{(e)}(m)=m+n=\sigma^{(e)}(n)$. Finally, e-aliquot sequences $n_{0}, n_{1}, n_{2}, \ldots$ where $n_{i+1}=\sigma^{(e)}\left(n_{i}\right)-n_{i}$ for $1=0,1,2, \ldots$ are studied in Section 4 .

2. THE DENSITY OF THE e-PERFECT NUMBERS.

By definition, $\sigma^{(e)}(1)=1$ and it is easy to see that $\sigma^{(e)}(n)$ is multiplicative. Therefore, since $\sigma^{(e)}(p)=p$ if $p$ is a prime, we see that $\sigma^{(e)}(m)=m$ if $m$ is squarefree.

Now suppose that $n$, as given by (1.1), is a powerful e-perfect number (so that $a_{i} \geqq 2$ for $i=1,2, \ldots, r$ and $\left.\sigma^{(e)}(n)=2 n\right)$. Then if $(m, n)=1$ and $m$ is squarefree then $\sigma^{(e)}(\mathrm{mn})=2 \mathrm{mn}$ so that $\mathrm{mn}$ is an e-perfect number. Therefore, if $x$ is a (fixed) positive number and $n_{1}<n_{2}<\ldots<n_{s}$ are the powerful e-perfect numbers which do not exceed $x$ then $E(x)$, the set of (a11) e-perfect numbers less than or equal to $x$, is given by $E(x)=\bigcup_{i=1}^{S} A_{i}$ where

$$
A_{i}=\left\{m n_{i}:\left(m, n_{i}\right)=1, m \leq x / n_{i} \text { and } m \text { is squarefree }\right\}
$$

Let $\mathrm{N}$ be a positive integer and let $\mathrm{X}$ be a positive real number. If $\mathrm{Q}(\mathrm{N}, \mathrm{X})$ is the number of positive, squarefree integers which do not exceed $X$ and which are relatively prime to $\mathrm{N}$, then $\mathrm{E}$. Cohen (Lemma 5.2 in [5]) has shown that

$$
Q(N, X)=B(N) \cdot X+0\left(\theta(N) \cdot X^{1 / 2}\right)
$$

where $\beta(N)=\left(\zeta(2) \prod_{p \mid N}(1+1 / p)\right)^{-1}$ and $\theta(N)$ is the number of squarefree divisors of $\mathrm{N}$. It is easy to see that $\theta(\mathrm{N})=\prod_{\mathrm{P} \mid \mathrm{N}} 2 . \zeta(\mathrm{k})$ is the Riemann Zeta function, so that $\zeta(2)=\pi^{2} / 6$, and the constant implied by the 0 -term is independent of $\mathrm{N}$ and $\mathrm{X}$.

If $Q(e, x)$ is the number of e-perfect numbers which do not exceed $x$ (so that $Q(e, x)$ is the cardinality of $E(x))$ it follows from (2.1) and (2.2) that

$$
Q(e, x)=x \sum_{i=1}^{s} \beta\left(n_{i}\right) / n_{i}+0\left(x^{1 / 2} \sum_{i=1}^{s} \theta\left(n_{i} / n_{i}^{1 / 2}\right)\right. \text {. }
$$

Therefore,

$$
Q(e, x) / x=\sum_{i=1}^{s} \beta\left(n_{i}\right) / n_{i}+0\left(x^{-1 / 2} \sum_{i=1}^{s} \theta\left(n_{i}\right) / n_{i}^{1 / 2}\right) .
$$

The following results concerning powerful numbers will be needed in what follows. Proofs may be found in Golomb [6].

LEMMA 1 . If $r_{1}<r_{2}<\ldots$ is the sequence of powerful numbers then $\sum_{i=1}^{\infty} 1 / r_{i}$ is convergent.

LEMMA 2. If $P(X)$ is the number of powerful numbers not exceeding $x$ then $P(x)<2.2 x^{1 / 2}$ for large $x$.

Now let $\varepsilon$ be a given positive number and let $\mathrm{P}_{i}$ denote the ith prime. There exists a positive integer $k$ such that

$$
2 / \mathrm{P}_{\mathrm{k}}<\varepsilon \cdot(2.2 \mathrm{~K})^{-1} / 3
$$

where $\mathrm{K}$ is the constant implied by the 0 -term in (2.3). 
Since there are only a finite number of powerful e-perfect numbers which are divisible by fewer that $k$ distinct primes (see Theorem 2.3 in [2]) there exists a positive integer $J$ such that if $n_{1}<n_{2}<\ldots$ is the sequence of powerful e-perfect numbers then for $a l 1 i>J n_{i}$ has at least $k$ distinct prime factors and $n_{i}$ has a prime factor, say $Q_{i}$, such that $Q_{i} \geqq P_{k}$. Since $n_{i}$ is powerful, $n_{i} 1 / 2 \geqq \pi p$ where the product is taken over the distinct prime factors of $n_{i}$, and it follows from (2.4) that if $i>J$ then

$$
\theta\left(\mathrm{n}_{\mathrm{i}}\right) / \mathrm{n}_{\mathbf{i}}^{\frac{1}{2}} \leqq \prod_{\mathrm{p} \mid \mathrm{n}_{\mathbf{i}}} 2 / \mathrm{p}<2 / \mathrm{Q}_{\mathbf{i}} \leqq 2 / \mathrm{P}_{\mathrm{k}}<\varepsilon \cdot(2.2 \mathrm{~K})^{-1} / 3 .
$$

Splitting the sum in the 0-term in (2.3) at $i=J$ (with $J$ held fixed) we can take $x$ large enough so that $x^{-1 / 2} \cdot K \cdot \sum_{i=1}^{J} \theta\left(n_{i}\right) / n_{i} 1 / 2<\varepsilon / 3$. At the same time, since every $n_{i}$ is powerful, we see from (2.5) and Lemma 2 that we can also take $x$ large enough so that

$$
\begin{aligned}
\mathrm{x}^{-1 / 2} \cdot \mathrm{K} \cdot \sum_{\mathrm{i}=\mathrm{J}+1}^{\mathrm{S}} \theta\left(\mathrm{n}_{\mathrm{i}}\right) / \mathrm{n}_{\mathrm{i}}^{1 / 2} & <\mathrm{x}^{-1 / 2} \cdot \mathrm{K} \cdot \sum_{\mathrm{i}=\mathrm{J}+1}^{\mathrm{s}} \varepsilon \cdot(2.2 \mathrm{~K})^{-1 / 3} \\
& <\mathrm{x}^{-1 / 2 \cdot \mathrm{P}(\mathrm{x}) \cdot \varepsilon \cdot(2.2)^{-1 / 3}<\varepsilon / 3 .}
\end{aligned}
$$

Finally, since $\beta\left(n_{i}\right)<1$ and every $n_{i}$ is powerful we see from Lemma 1 that $\sum_{i=1}^{\infty} B\left(n_{i}\right) / n_{i}$ is convergent. (This series may be finite since whether or not the set of powerful e-perfect numbers is finite or infinite is an open question). It follows that we can take $x$ (and consequently s) large enough so that the tail of this series is less that $\varepsilon / 3$. Therefore, from (2.3) we have for all large values of $x$,

$$
\left|Q(e, x) / x-\sum_{i=1}^{\infty} B\left(n_{i}\right) / n_{i}\right|<\varepsilon
$$

We have proved

THEOREM 1. Let $Q(e, x)$ denote the number of e-perfect numbers which do not exceed $x$ and let $n_{1}<n_{2}<n_{3}<\ldots$ be the sequence of powerful numbers. Then

$$
\lim _{x \rightarrow \infty} Q(e, x) / x=\sum_{i=1}^{\infty} B\left(n_{i}\right) / n_{i}=C
$$

where $B(n)=6 \pi^{-2} \prod_{p \mid n}(1+1 / p)^{-1}$. Correct to ten decimal places, $C=.0086941940$.

(There are eight powerful e-perfect numbers less than $10^{10}$ : $36 ; 1800 ; 2700$; 17,$424 ; 1,306,800 ; 4,769,856 ; 238,492,800 ; 357,739,200$. The approximate value of $\mathrm{C}$ given above was calculated using these eight numbers).

The "theoretical" density of the e-perfect numbers as given in Theorem 1 agrees very nicely with the following exact computational results: $Q\left(e, 10^{5}\right) / 10^{5}=.00871$; $Q\left(e, 10^{6}\right) / 10^{6}=.008690 ; Q\left(e, 10^{7}\right) / 10^{7}=.0086940 ; Q\left(e, 10^{8}\right) 10^{8}=.00869417$.

3. EXPONENTIALLY AMICABLE NUMBERS.

We shall say that $m$ and $n$ are exponentially amicable (or e-amicable) numbers if

$$
\sigma^{(e)}(m)=m+n=\sigma^{(e)}(n) .
$$


LEMMA 3. If $(m ; n)$ is an e-amicable pair and $p$ is a prime, then $p / m$ if and only if $\mathrm{p} / \mathrm{n}$.

PROOF. Suppose that $p^{a}|| m$ where $a \geqq 1$. Then $p / \sigma^{(e)}(m)$ since $p / \sigma^{(e)}\left(p^{a}\right)$ and $\sigma^{(e)}$ is a multiplicative function. It is now obvious from (3.1) that $p / n$. By the same argument, if $\mathrm{p} / \mathrm{n}$ then $\mathrm{p} / \mathrm{m}$.

COROLLARY 3.1. If $(m ; n)$ is an e-amicable pair then $m=n(m o d 2)$.

If $(m ; n)$ is an e-amicable pair and there is no prime $p$ such that $p|| m$ and $p / \mid n$ we shall say that $m$ and $n$ are primitive e-amicable numbers. It is easy to see that if $(m ; n)$ is a primitive e-amicable pair and $r$ is a squarefree positive integer such that $(m, r)=1$, then $(r m ; r n)$ is an amicable pair.

A search was made for all primitive e-amicable pairs $(m ; n)$ such that $m<n$ and $\mathrm{m}<10^{7}$. The search required about 1.5 hours on the CDC CYBER 750 and three pairs were found. They are as follows: $\left(2^{2} 3^{2} 7 \cdot 19^{2} ; 2^{2} 3^{3} 7^{2} 19\right) ;\left(2^{2} 3^{2} 7 \cdot 61^{2} ; 2^{2} 3^{4} 7^{2} 61\right)$; $\left(2^{3} 3^{2} 5^{2} 7 \cdot 19^{2} ; 2^{3} 3^{3} 5^{2} 7^{2} 19\right)$.

This list suggests the following questions. Are there any odd e-amicable numbers? Are there any powerful e-amicable numbers? Is every e-amicable number divisible by at least four distinct primes? (It is easy to show that every e-amicable number has at least three different prime factors).

The following result can sometimes be used to generate new e-amicable pairs from known pairs.

THEOREM 2. Suppose that $(a M ; a N)$ is an e-amicable pair such that $(a, M)=(a, N)=1$. If $(b, M)=(b, N)=1$ and $\sigma^{(e)}(a) / a=\sigma^{(e)}(b) / b$ then $(b M, b N)$ is an e-amicable pair. PROOF. $\sigma^{(e)}(b M)=\sigma^{(e)}(b) \cdot \sigma^{(e)}(M)=a^{-1} b \sigma^{(e)}(a) \cdot \sigma^{(e)}(M)=a^{-1} b \sigma^{(e)}(a M)=\sigma$ $a^{-1} b(a M+a N)=b M+b N$. Similarly, $\sigma^{(e)}(b N)=b M+b N$.

The results of a computer search for powerful numbers a and $b$ such that $4 \leqq a<b \leqq 10000$ and $\sigma^{(e)}(a) / a=\sigma^{(e)}(b) / b$ are given in Table I.

TABLE I

\begin{tabular}{|l|c|c|}
\hline$\sigma^{(e)}(a) / a$ & $a$ & $b$ \\
\hline $3 / 2$ & $2^{2}$ & $2^{3} 5^{2}$ or $2^{4} 11^{2}$ \\
\hline $4 / 3$ & $3^{2}$ & $3^{3} 5^{2}$ \\
\hline 2 & $2^{2} 3^{2}$ & $2^{3} 3^{5} 5^{2}$ or $2^{2} 3^{3} 5^{2}$ \\
\hline $39 / 32$ & $2^{6}$ & $2^{7} 5^{2}$ \\
\hline $5 / 3$ & $2^{3} 3^{2}$ & $2^{2} 3^{3}$ or $2^{3} 3^{3} 5^{2}$ \\
\hline $12 / 7$ & $2^{2} 7^{2}$ & $2^{3} 5^{2} 7^{2}$ \\
\hline $65 / 48$ & $2^{7} 3^{2}$ & $2^{6} 3^{3}$ \\
\hline $40 / 21$ & $2^{3} 3^{2} 7^{2}$ & $2^{2} 3^{3} 7^{2}$ \\
\hline
\end{tabular}


EXAMPLE. Since $\left(2^{2} \cdot 3^{2} \cdot 7 \cdot 19^{2} ; 2^{2} \cdot 3^{3} \cdot 7^{2} \cdot 19\right)$ is an e-amicable pair and since $\sigma^{(\mathrm{e})}\left(2^{2}\right) / 2^{2}=\sigma^{(\mathrm{e})}\left(2^{4} \cdot 11^{2}\right) / 2^{4} \cdot 11^{2}$ it follows from Theorem 2 that $\left(2^{4} \cdot 11^{2} \cdot 3^{2} \cdot 7 \cdot 19^{2} ; 2^{4} \cdot 11^{2} \cdot 3^{3} \cdot 7^{2} \cdot 19\right)$ is an e-amicable pair.

4. EXPONENTIAL ALIQUOT SEQUENCES.

The function $s^{(e)}$ is defined by $s^{(e)}(n)=\sigma^{(e)}(n)-n$, the sum of the exponential aliquot divisors of $n$. $s^{(e)}(1)=s^{(e)}(r)=0$ for every squarefree number $r$ and we define $s^{(e)}(0)=0$. A t-tuple of distinct natural numbers $\left(n_{0} ; n_{1} ; \ldots ; n_{t-1}\right)$ with $n_{i}=s^{(e)}\left(n_{i-1}\right)$ for $i=1,2, \ldots, t-1$ and $s^{(e)}\left(n_{t-1}\right)=n_{0}$ is called an exponential $t$-cycle. An exponential 1-cycle is an e-perfect number and an exponential 2-cycle is an e-amicable pair. A search was made for all exponential $t$-cycles with smallest member not exceeding $10^{7}$. None with $t>2$ was found.

The exponential aliquot sequence (or e-aliquot sequence) $\left\{n_{i}\right\}$ with leader $n$ is defined by $n_{0}=n, n_{1}=s^{(e)}\left(n_{0}\right), n_{i}=s^{(e)}\left(n_{i-1}\right), \ldots$. Such a sequence is said to be terminating if $n_{k}$ is squarefree for some index $k$ (so that $n_{1}=0$ for $1>k$ ). An exponential aliquot sequence is said to be periodic if there is an index $k$ such that $\left(n_{k} ; n_{k+1} ; \ldots ; n_{k+t-1}\right)$ is an exponential $t$-cycle. An e-aliquot sequence which is neither terminating nor periodic is unbounded.

An investigation was made of all aliquot sequences with leader $n \leq 10^{7}$. About 2.3 hours of computer time was required. 9,896,235 were found to be terminating and 103,765 were periodic (103,694 ended in 1-cycles and 71 ended in 2-cycles).

The fact that the first ten million exponential aliquot sequences are bounded might tempt one to conjecture that the set of unbounded e-aliquot sequences is empty. However, the following theorem shows that e-aliquot sequences exist which contain arbitrarily long strings of monotonically increasing terms. Therefore, whether or not unbounded e-aliquot sequences exist would seem to be a very open and difficult question.

THEOREM 3. Let $\mathrm{N}$ be a positive integer which exceeds 2 . Then there exist infinitely many exponential aliquot sequences such that $n_{0}<n_{1}<n_{2}<\ldots<n_{N-2}$.

PROOF. Let $q_{1}, q_{2}, \ldots, q_{N}$ be a sequence of $N$ primes such that $q_{1}=2, q_{2}=3$ and $q_{i}^{2} \mid\left(q_{i+1}+1\right)$ for $i=2,3, \ldots, N-1$. (Infinitely many such sequences exist since, by Dirichlet's theorem, the arithmetic progression $\mathrm{aq}_{1}^{2}-1$ contains an infinite number of primes.) We shall write $q_{i+1}+1=k_{i} q_{i}^{2}$.

Now let $n_{0}, n_{1}, n_{2}, \ldots$ be the exponential aliquot sequence with leader $n_{0}$ given by $\mathrm{n}_{0}=\mathrm{q}_{1}^{2} \mathrm{q}_{2}^{2} \ldots \mathrm{q}_{\mathrm{N}}^{2}$. Then

$$
\begin{aligned}
\sigma^{(e)}\left(n_{0}\right) & =\prod_{i=1}^{N}\left(q_{i}+q_{i}^{2}\right)=3 \cdot q_{1} q_{2} \cdots q_{N} \cdot \prod_{i=2}^{N}\left(1+q_{1}\right) \\
& =3 \cdot q_{1} q_{2} \cdots q_{N} \cdot \prod_{i=1}^{N-1} k_{i} q_{i}^{2},
\end{aligned}
$$

and

$$
n_{1}=\sigma^{(e)}\left(n_{0}\right)-n_{0}=\left(3 \cdot q_{1} q_{2} \cdots q_{N} \cdot k_{1} \cdots k_{N-1}-q_{N}^{2}\right) \cdot \prod_{i=1}^{N-1} q_{i}^{2} .
$$

Therefore, $n_{1}=M_{1} \prod_{i=1}^{N-1} q_{1}^{2}$ where $\left(M_{1}, q_{i}\right)=1$ for $i=1,2, \ldots, N-1$. 
Since $n_{0} / 36$ is not squarefree, $n_{1}=\sigma^{(e)}\left(n_{0}\right)-n_{0}=\sigma^{(e)}(36) \cdot \sigma^{(e)}\left(n_{0} / 36\right)-n_{0}$ $=72 \cdot \sigma^{(e)}\left(n_{0} / 36\right)-n_{0}>72 \cdot n_{0} / 36-n_{0}=n_{0}$.

Similarly, we find that for $k=2,3, \ldots, N-2$

$$
n_{k}=M_{k} \prod_{i=1}^{N-k} q_{i}^{2} \text { where }\left(M_{k}, q_{i}\right)=1 \text { for } i=1,2, \ldots, N-k
$$

and

$$
\begin{aligned}
n_{k} & =\sigma^{(e)}\left(n_{k-1}\right)-n_{k-1}=\sigma^{(e)}(36) \cdot \sigma^{(e)}\left(n_{k-1} / 36\right)-n_{k-1} \\
& >72 \cdot n_{k-1} / 36-n_{k-1}=n_{k-1} .
\end{aligned}
$$

Therefore, $\mathrm{n}_{\mathrm{o}}<\mathrm{n}_{1}<\ldots<\mathrm{n}_{\mathrm{N}-2}$.

REMARK 1. $\mathrm{n}_{\mathrm{N}-2}=36 \mathrm{M}-2$ where $\left(6, \mathrm{M}_{\mathrm{N}-2}\right)=1$. If $\mathrm{M}_{\mathrm{N}-2}$ is not squarefree, then $\mathrm{n}_{\mathrm{N}-1}=72 \cdot \sigma^{(\mathrm{e})}\left(\mathrm{M}_{\mathrm{N}-2}\right)-36 \mathrm{M}_{\mathrm{N}-2}>72 \mathrm{M}_{\mathrm{N}-2}-36 \mathrm{M}_{\mathrm{N}-2}=36 \mathrm{M}_{\mathrm{N}-2}=\mathrm{n}_{\mathrm{N}-2}$.

REMARK 2. The proof of Theorem 3 is modeled on that of Theorem 2.1 in [7].

Our next objective is to determine $M\left(\sigma^{(e)}(n) / n\right)$, the mean value of $\sigma^{(e)}(n) / n$. The mean value of an arithmetic function $f$ is defined by $M(f)=\lim _{N \rightarrow \infty} N^{-1} \sum_{n}^{N} f(n)$.

We shall need the following lemma due to van der Corput (See Theorem A in [8].)

LEMMA 4. If $f$ and $h$ are arithmetic functions such that $f(n)=\sum_{d} h(d)$ and $\sum_{n=1}^{\infty} h(n) / n$ is absolutely convergent then $M(f)=\sum_{n=1}^{\infty} h(n) / n$.

We wish to apply this lemma to the function $f(n)=\sigma^{(e)}(n) / n$. By the Moebius inversion formula, $h(n)=\sum_{d} \mu(n / d) \sigma^{(e)}(d) / d . \quad h$ is multiplicative and $h(1)=1$. If $p$ is a prime and a is a positive integer then $h\left(p^{a}\right)=\sigma^{(e)}\left(p^{a}\right) / p^{a}-\sigma^{(e)}\left(p^{a-1}\right) / p^{a-1}$. If $a<6$ it is easy to verify that $\left|h\left(p^{a}\right)\right|<p^{-a / 4}$. (For example, $\left|\mathrm{h}\left(\mathrm{p}^{3}\right)\right|=\mathrm{p}^{-1}-\mathrm{p}^{-2}<\mathrm{p}^{-1}<\mathrm{p}^{-3 / 4}$.) Suppose that $\mathrm{a} \geq 6$. Then $\left|\mathrm{h}\left(\mathrm{p}^{\mathrm{a}}\right)\right|=\sigma^{(\mathrm{e})}\left(\mathrm{p}^{\mathrm{a}}\right) / \mathrm{p}^{\mathrm{a}}-\sigma^{(\mathrm{e})}\left(\mathrm{p}^{\mathrm{a}-1}\right) / \mathrm{p}^{\mathrm{a}-1}$ or $\left|\mathrm{h}\left(\mathrm{p}^{\mathrm{a}}\right)\right|=\sigma^{(\mathrm{e})}\left(\mathrm{p}^{\mathrm{a}-1}\right) / \mathrm{p}^{\mathrm{a}-1}-\sigma^{(\mathrm{e})}\left(\mathrm{p}^{\mathrm{a}}\right) / \mathrm{p}^{\mathrm{a}}$. Since $\sigma^{(e)}\left(p^{m}\right) / p^{m}<1+p /(p-1) p^{m / 2}$ (see [2] or [4]) and $\sigma^{(e)}\left(p^{b}\right) / p^{b} \geqq 1$, $\left|h\left(p^{a}\right)\right|<p /(p-1) p^{(a-1) / 2}$. Since $a \geq 6$ it follows easily that $\left|h\left(p^{a}\right)\right|<p^{-a / 4}$. Since $h$ is multiplicative, $|h(n)| \leqq n^{-1 / 4}$ for every positive integer $n$. It follows that $\sum_{n=1}^{\infty} h(n) / n$ is absolutely convergent so that Lemma 4 applies if $f(n)=\sigma^{(e)}(n) / n$. From Theorem 286 in [9] we have

$$
\begin{aligned}
\sum_{n=1}^{\infty} h(n) / n & =\prod_{p}\left\{1+h(p) / p+h\left(p^{2}\right) / p^{2}+\ldots\right\} \\
& =\prod_{p}\left\{1+p^{-1}\left(\sigma^{(e)}(p) / p-1\right)+p^{-2}\left(\sigma^{(e)}\left(p^{2}\right) / p^{2}-\sigma^{(e)}(p) / p\right)+\ldots\right\} \\
& =\prod_{p}\left\{\sum_{j=0}^{\infty} \sigma^{(e)}\left(p^{j}\right) / p^{2 j}-p^{-1} \sum_{j=0}^{\infty} \sigma^{(e)}\left(p^{j}\right) / p^{2 j}\right\} \\
& =\prod_{p}\left\{\left(1-p^{-1}\right) \sum_{j=0}^{\infty} \sigma^{(e)}\left(p^{j}\right) / p^{2 j}\right\} .
\end{aligned}
$$

Now the last infinite series can be "split up" by first taking all the terms with numerator $p^{j}$ to form the series $\sum_{j=0}^{\infty} p^{j} / p^{2 j}=\sum_{j=0}^{\infty} 1 / p^{j} ;$ then taking the remaining 
terms with numerators $p$ to form the series $\sum_{j=2}^{\infty} p / p_{\infty}^{2 j}=p^{-3} \sum_{j=0}^{\infty}\left(p^{-2}\right)^{j}$; then taking the terms with numerators $p^{2}$ to form the series $\sum_{j=2}^{\infty} p^{2} / p^{4 j}=p^{j=6} \sum_{j=0}^{\infty}\left(p^{-4}\right)^{j}$; then taking the terms with numerators $p^{3}$ to form the series $\sum_{j=2}^{\infty} p^{3} / p^{6 j}=p^{j=0} \sum_{j=0}^{\infty}\left(p^{-6}\right)^{j}$; etc. It follows that

$$
\begin{aligned}
\sum_{n=1}^{\infty} h(n) / n & =\prod_{p}\left\{( 1 - p ^ { - 1 } ) \left(\left(1-p^{-1}\right)^{-1}+p^{-3}\left(1-p^{-2}\right)^{-1}\right.\right. \\
& \left.\left.+p^{-6}\left(1-p^{-4}\right)^{-1}+p^{-9}\left(1-p^{-6}\right)^{-1}+\ldots\right)\right\} \\
& =\prod_{p}\left\{( 1 - p ^ { - 1 } ) \left(\left(1-p^{-1}\right)^{-1}+\left(p^{3}-p\right)^{-1}+\left(p^{6}-p^{2}\right)^{-1}\right.\right. \\
& \left.\left.+\left(p^{9}-p^{3}\right)^{-1}+\ldots\right)\right\} \\
& =\prod_{p}\left\{1+\left(1-p^{-1}\right) \sum_{j=1}^{\infty}\left(p^{3 j}-p^{j}\right)^{-1}\right\} .
\end{aligned}
$$

From Lemma 4 we have

THEOREM 4. $M\left(\sigma^{(e)}(n) / n\right)=\prod_{p}\left\{1+\left(1-p^{-1}\right) \cdot \sum_{j=1}^{\infty}\left(p^{3 j}-p^{j}\right)^{-1}\right\}=c$.

Correct to 6 decimal places, $\mathrm{C}=1.136571$.

(This approximate value of $C$ was calculated using all primes less than $10^{6}$ in the infinite product.)

Since $s^{(e)}(n)=\sigma^{(e)}(n)-n$ we have

COROLLARY 4.1. $M\left(s^{(e)}(n) / n\right)=.136571$.

Finally, since $n_{i+1} / n_{i}=s^{(e)}\left(n_{i}\right) / n_{i}$ we see that, in some sense, the average value of the ratio of two consecutive non-zero terms of an e-aliquot sequence is about .136571 .

\section{REFERENCES}

1. SUBBARAO, M.V. On some arithmetic convolutions, The Theory of Arithmetic Functions, Lecture Notes in Mathematics 251 (1972), Springer-Verlag, New York, 247-271.

2. Straus, E.G. and SUbBaraO, M.V. On exponential divisors, Duke Math. J. 41 (1974), 465-471.

3. FABRYKOWSKI, J. and SUBBARAO, M.V. On e-perfect numbers and a conjecture of Straus and Subbarao, Congressus Numerantium 52 (1986), 79-90.

4. SUBBARAO, M.V., HARDY, G.E. and AIELLO, W. On the existence of e-multiperfect numbers, The Fibonacci Quarterly 25 (1987), 65-71.

5. COHEN, E. Arithmetical functions associated with the unitary divisors of an integer, Math. Zeit. 프 (1960), 66-80.

6. GOLOMB, S.W. Powerful Numbers, Amer. Math. Monthly 77 (1970), 848-855.

7. te RIELE, H.J.J. A theoretical and computational study of generalized aliquot sequences, Mathematical Centre Tracts 74 (1976), Amsterdam.

8. COHEN, E. ARITHMATICAL NOTES, I. On a theorem of van der Corput, Proc. A.M.S. $12(1961), 214-217$.

9. HARDY, G.H. and WRIGHT, E.M. An Introduction to the Theory of Numbers (Fourth Edition) Oxford University Press, New York, 1960. 


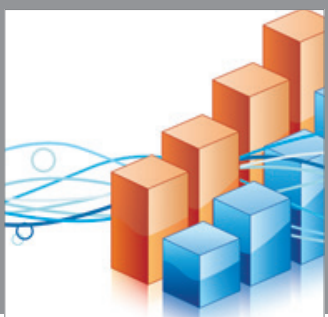

Advances in

Operations Research

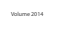

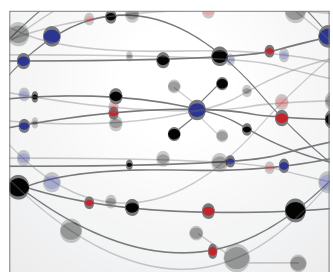

\section{The Scientific} World Journal
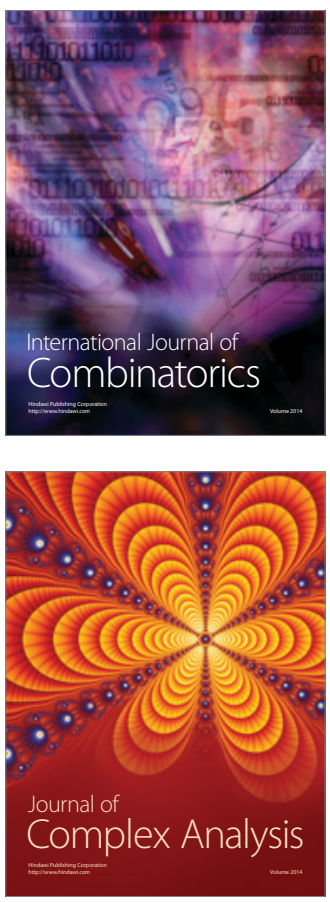

International Journal of

Mathematics and

Mathematical

Sciences
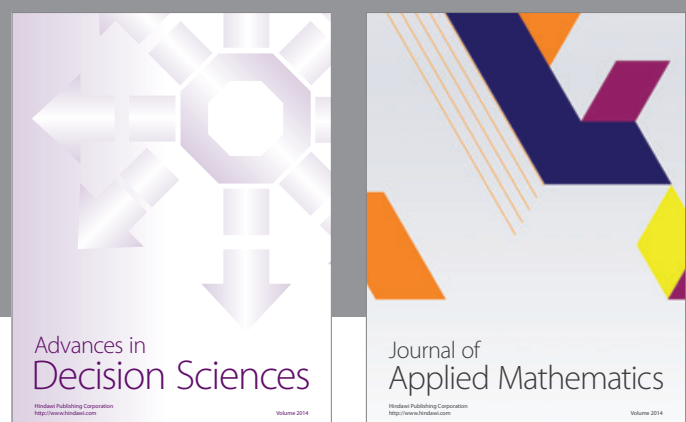

Journal of

Applied Mathematics
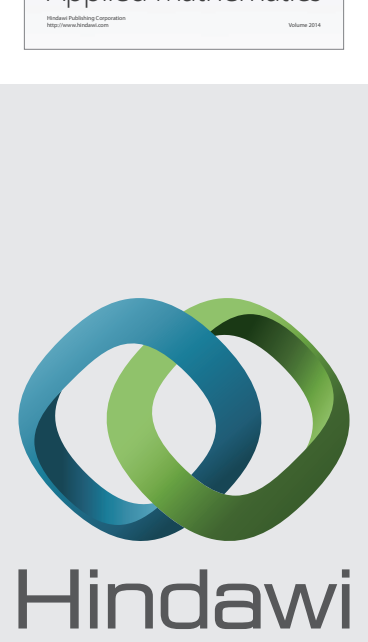

Submit your manuscripts at http://www.hindawi.com
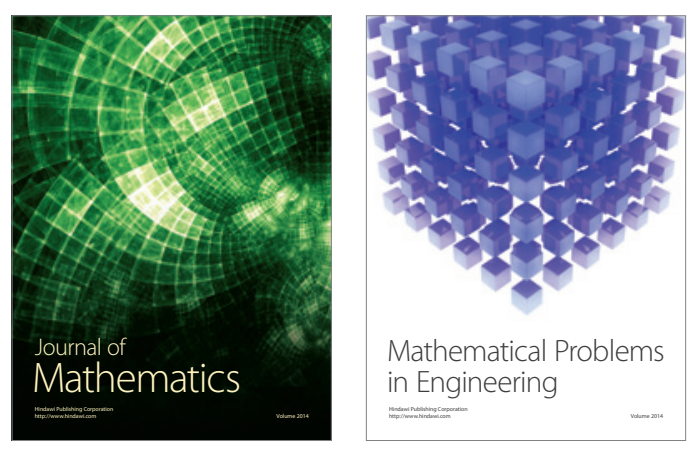

Mathematical Problems in Engineering
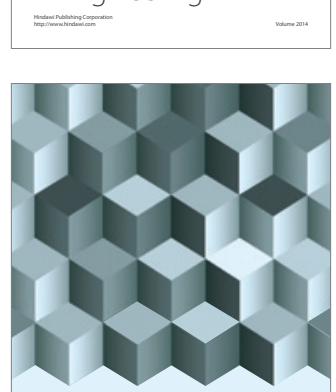

Journal of

Function Spaces
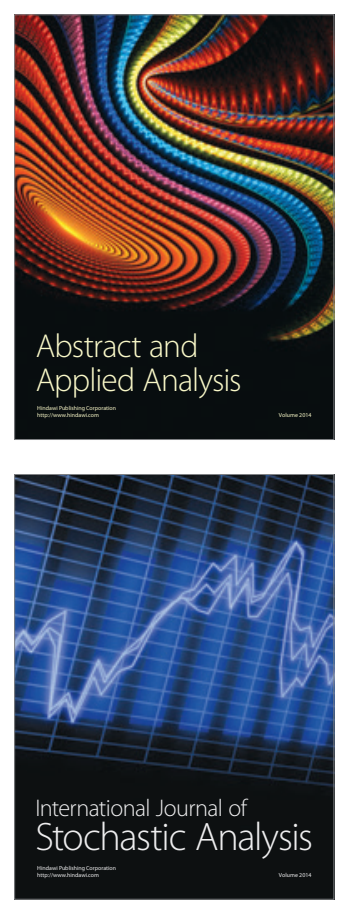

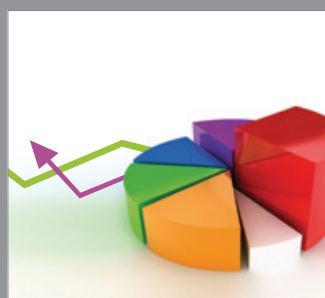

ournal of

Probability and Statistics

Promensencen
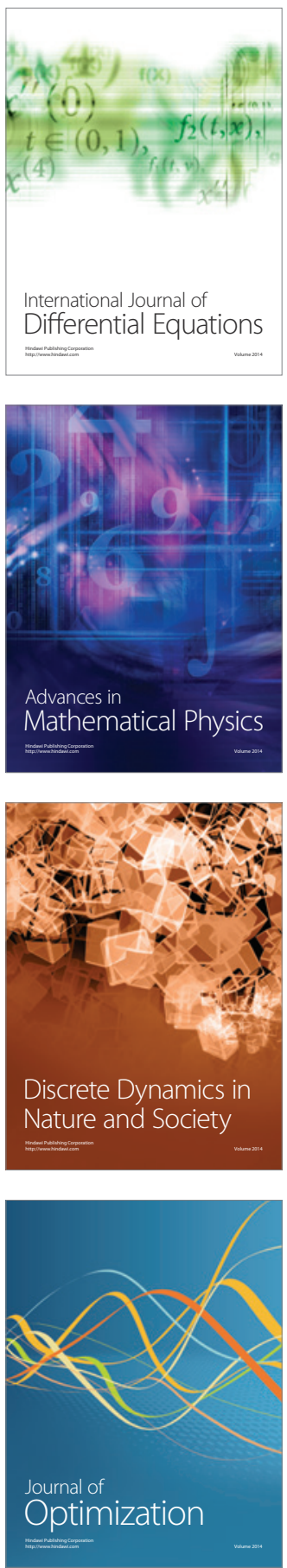